\title{
Effects of levothyroxine therapy on bone mineral density and bone turnover markers in premenopausal women with thyroid cancer after thyroidectomy
}

\author{
Duan Bin-Hong, Du Fu-Man, Liu Yu, Wang Xu-Ping, Bian Bing-Feng \\ Department of Endocrinology, Heilongjiang Provincial Hospital, Harbin Heilongjiang Province, China
}

\begin{abstract}
Introduction: We investigated the impact of long-term levothyroxine (LT4) treatment on bone mineral density (BMD) and bone turnover markers (BTMs) in premenopausal women with differentiated thyroid cancer (DTC) after thyroidectomy.

Material and methods: Sixty-five premenopausal women who received LT4 therapy at least one year (range, 1.5-9.0 years) after thyroidectomy for DTC and 50 premenopausal women without thyroid diseases were enrolled in this study. We measured the Z-scores of lumbar and hip BMD, serum free triiodothyronine (FT3), free thyroxine (FT4), thyroid-stimulating hormone (TSH), intact parathyroid hormone (iPTH), N-terminal propeptide of type $1 \mathrm{~N}$ procollagen (P1NP), C-terminal telopeptide of type 1 collagen (CTX-1), calcium (Ca), phosphorus (P), vitamin D3, and alkaline phosphatase (ALP) in all participants.

Results: In DTC subjects, serum TSH levels were lower, and serum FT4, P1NP, CTX-1, and ALP levels were higher compared with controls. The prevalence of low BMD was higher in DTC subjects than in controls. Multivariate logistic regression analysis showed that serum TSH levels were negatively associated with CTX-1 and ALP.

Conclusions: We found a high prevalence of low BMD among premenopausal women who received long-term LT4 therapy for DTC after thyroidectomy. Long-term TSH suppression therapy was a significant risk factor for decreased bone strength, mainly by increasing bone turnover. (Endokrynol Pol 2020; 71 (1): 15-20)
\end{abstract}

Key words: levothyroxine; bone mineral density; women; bone turnover markers; thyroid cancer; thyroidectomy

\section{Introduction}

Thyroid cancer is one of the most common endocrine malignancies. The incidence of DTC has increased substantially in the last four decades [1-3]. Most of the thyroid cancer raised from thyroid follicular epithelial cells, a well-differentiated cancer, have an excellent prognosis, with reported 10-year survival rates reaching $90 \%[4,5]$. If the treatment according to the current guideline is given, cases of recurrence and persistence are rare [6-8]. After diagnosis, patients with thyroid cancer will generally undergo total or near total thyroidectomy. These patients, after thyroidectomy, will be treated with long-term LT4 for TSH suppression in order to inhibit cancer recurrence. For the first two years the serum TSH levels are required to be $<0.1 \mathrm{mIU} / \mathrm{L}$. If there was no recurrence in the first year or if the patients were low-risk recurrent, the serum TSH levels can be kept with $0.1-0.5 \mathrm{mIU} / \mathrm{L}[9,10]$. This means that most of the patients receiving TSH suppression treatment are in a state of hyperthyroidism or subclinical hyper- thyroidism, with high risk of adverse effects, including cardiovascular or skeletal outcomes [11-13].

Thyroid hormones are considered essential for bone metabolism in adults. Thyroid hormones have important effects on the balance of skeletal development and bone linear growth, which maintain bone mass and strength [14, 15]. Hyperthyroidism causes high bone turnover and shortening of the normal remodelling cycle, leading to decreased BMD and bone strength. Hyperthyroidism and subclinical hyperthyroidism are all well known risk factors for osteoporosis or osteopaenia [16-18]. The American Thyroid Association (ATA) recommend treating patients with levels of TSH less than $0.1 \mathrm{mIU} / \mathrm{L}$ if they are older than 65 years or suffer from cardiovascular diseases or osteoporosis [19]. However, the effect of long-term TSH suppressive therapy on BMD and BTMs in patients with DTC after thyroidectomy is still unclear [20-22], and most of these studies involved postmenopausal women, who have a higher risk of osteoporosis. In this study, we try to investigate the impact of long-term LT4 treatment on 
BMD and BTMs in premenopausal women with DTC after thyroidectomy.

\section{Material and methods}

\section{Subjects}

From May 2018 to April 2019, we randomly selected 65 premenopausal women (aged 32 to 45 years) with DTC from the Endocrinology Clinic of Heilongjiang Provincial Hospital, Harbin, China. All participants had undergone total or near total thyroidectomy and received LT4 therapy for at least one year in order to maintain a serum TSH concentration between 0.1 and $0.5 \mathrm{mIU} / \mathrm{L}$. Mean duration of levothyroxine treatment was $5.25 \pm 3.14$ years (range, 1.5-9.0 years). The mean dosage of levothyroxine was $133.6 \mu \mathrm{g}$ (range, 25-150 $\mu \mathrm{g} /$ day). At the same time, we randomly selected 50 premenopausal women without history of thyroid diseases (aged 29 to 45 years) from a physical examination centre at the same hospital. We excluded participants with a history of alcohol abuse, asthma, severe renal or hepatic diseases, hyperthyroidism, hyperparathyroidism, hypoparathyroidism, hypercalcaemia, chronic use of corticosteroid, severe gastrointestinal diseases, malignant tumour, diabetes mellitus, severe cardiac diseases, rheumatoid arthritis, and other rheumatic-inflammatory diseases from this study. Participants who underwent any treatment of osteoporosis or osteopaenia like selective oestrogen receptor modulators, bisphosphonate, and calcitonin were also excluded from this study.

\section{Definitions}

Premenopausal women were those aged $18-45$ years with normal menstrual cycle. Low BMD in premenopausal women is diagnosed according to the diagnostic criteria of the World Health Organisation (WHO) [23]. Lumbar or hip BMD by DXA; $\mathrm{Z}$ score less than or equal to -2.0 .

\section{Laboratory measurements}

The Z-scores of BMD of lumbar spine (L2-L4) and hip (femoral neck, ward triangle, trochanter, total hip) were measured using a dual-energy X-ray absorptiometry (DXA) instrument (Hologic, Inc., Bedford, MA, USA). The coefficient of variation of BMD in our hospital is $1.40 \%$ in the lumbar spine and $1.50 \%$ in the hip. Participants were measured on the same instrument, using the same scan speed and doctor. Fasting blood samples were obtained at least eight-hour overnight fast from all subjects. Serum CTX-1 levels were measured by enzyme-linked immunosorbent assay (ELISA). The sensitivity was $1.224 \mathrm{ng} / \mathrm{mL}$, the intra-assay coefficient of variability (CV\%) was 10.05, and the inter-assay CV\% was 10.34 . Serum P1NP was measured by radioimmunoassay (RIA). The sensitivity was $0.842 \mathrm{ug} / \mathrm{L}$, the intra-assay coefficient of CV\% was 5.65, and the inter-assay CV\% was 5.484. Serum FT3, FT4, TSH, $\mathrm{iPTH}$, and vitamin D3 levels were measured using super sensitive electrochemiluminescence immunoassays (Roche Diagnostics, Mannheim, Germany). The normal reference range for serum TSH is $0.35-4.94 \mathrm{mIU} / \mathrm{L}$, for FT3 is $2.60-5.70 \mathrm{pmol} / \mathrm{L}$, and for FT4 is 9.0-19.0 pmol/L. Serum Ca, P, and ALP levels were measured by automatic biochemical analyser (Hitachi, Ltd., Tokyo, Japan). The measurement of serum CTX-1, P1NP, FT3, FT4, TSH, iPTH, and vitamin D3 levels was performed at the Centre of Special Radioimmunoassay in Heilongiiang Provincial Hospital. The measurement of serum Ca, P, and ALP was performed at the Department of Experimental Diagnosis in Heilongjiang Provincial Hospital.

\section{Medical ethics approval}

This study was approved by the Medical Ethics Committee of Heilongjiang Provincial Hospital. Written consent to participate in this study was obtained from all participants. Only those who signed the consent form were enrolled in the study. All study protocols were carried out in accordance with the declaration of Helsinki [24].

\section{Statistical analysis}

Statistical analysis was performed using IBM SPSS version 20.0 (IBM Corp., Armonk, NY, USA). Categorical data were evaluated in terms of frequency and percentage, and continuous data were presented as means \pm standard deviation. Chi-square test was used to compare the prevalence of osteoporosis and osteopaenia between two groups. The variables were compared using twosample $t$-test. Pearson correlation analysis was used to estimate the relationship of thyroid hormones with Z-scores of BMD and BTMs. The odds ratios (ORs) and $95 \%$ confidence intervals (CIs) for FT3, FT4, and TSH, in association with Z-scores of BMD, iPTH, P1NP, CTX-1, Ca, P, vitamin D3, and ALP were analysed using multivariate logistic regression. The $\mathrm{p}$-value $<0.05$ was considered as statistically significant.

\section{Results}

\section{Laboratory characteristics of the two study groups}

The descriptive statistics of the characteristics were shown in Table 1. In DTC subjects, serum TSH levels were lower, serum FT4, P1NP, CTX-1, and ALP levels were higher compared with controls. There were no significant differences in age, $\mathrm{Z}$-scores of $\mathrm{BMD}$, serum FT3, iPTH, Ca, P, and vitamin D3 levels between the two study groups.

\section{Prevalence of low BMD in the study groups}

The prevalence of low BMD was higher in the DTC group than in the control group. A comparison of prevalence of low BMD between the two groups is shown in Table 2.

\section{The relationship of serum thyroid hormone levels with Z-scores of BMD and BTMs}

We found no obvious associations between serum FT3, FT4, and TSH levels and Z-scores of BMD, iPTH, P1NP, CTX-1, Ca, P, vitamin D3, and ALP in controls. Similarly, there were no significant associations between serum FT3, FT4 levels, and the above parameters in the DTC group. Serum TSH levels were negatively associated with CTX-1 and ALP. There were no associations of serum TSH levels with Z-scores of BMD and other BTMs. Correlation analysis between serum thyroid hormone levels and Z-scores of BMD and BTMs in the DTC group are shown in Table 3.

\section{Analysing the relationship of serum thyroid hormones levels and Z-scores of BMD and BTMs using multivariate logistic regression}

After adjusting for age, serum TSH levels negatively associated with CTX-1 and ALP, not with Z-scores of $\mathrm{BMD}$, iPTH, P1NP, Ca, P, and vitamin D3. Serum FT3 and FT4 levels did not associate with Z-scores of BMD 
Table 1. Comparison of characteristics between the group with differentiated thyroid cancer (DTC) and the control group

\begin{tabular}{lccc}
\hline Characteristics & Control group $(\mathbf{n}=\mathbf{5 0})$ & DTC group $(\mathbf{n}=\mathbf{6 5})$ & $\mathbf{p}$-value \\
\hline FT3 [pmol/L] & $4.12 \pm 1.03$ & $4.15 \pm 1.09$ & $t=0.15, \mathrm{p}=0.881$ \\
\hline FT4 [pmol/L] & $13.12 \pm 3.56$ & $14.59 \pm 4.07^{*}$ & $t=2.026, \mathrm{p}=0.045$ \\
\hline TSH [mlU/L] & $1.65 \pm 1.45$ & $0.35 \pm 0.14^{*}$ & $t=-7.194, \mathrm{p}<0.001$ \\
\hline iPTH [m/U/L] & $15.75 \pm 6.48$ & $16.15 \pm 4.22$ & $t=0.399, \mathrm{p}=0.69$ \\
\hline $\mathrm{Ca}[\mathrm{mmol} / \mathrm{L}]$ & $2.46 \pm 0.34$ & $2.44 \pm 0.26$ & $t=-0.358, \mathrm{p}=0.721$ \\
\hline $\mathrm{P}[\mathrm{mmol} / \mathrm{L}]$ & $1.12 \pm 0.25$ & $1.15 \pm 0.36$ & $t=0.503, \mathrm{p}=0.616$ \\
\hline ALP [IU/L] & $95.15 \pm 21.26$ & $104.13 \pm 18.43^{*}$ & $t=2.422, \mathrm{p}=0.017$ \\
\hline CTX-1 [ng/mL] & $0.24 \pm 0.13$ & $0.31 \pm 0.16^{*}$ & $t=2.519, \mathrm{p}=0.013$ \\
\hline P1NP [ug/L] & $24.40 \pm 11.41$ & $29.87 \pm 15.53^{*}$ & $t=2.093, \mathrm{p}=0.039$ \\
\hline Vitamin D3 [ug/L] & $19.40 \pm 3.22$ & $18.8 \pm 3.47$ & $t=-0.948, \mathrm{p}=0.345$ \\
\hline Mean Z-scores of lumbar BMD & $-0.91 \pm 0.98$ & $-0.94 \pm 1.32$ & $t=-0.1346, \mathrm{p}=0.893$ \\
\hline Mean Z-scores of hip BMD & $-0.92 \pm 1.09$ & $-0.91 \pm 1.76$ & $t=0.073, \mathrm{p}=0.942$ \\
\hline
\end{tabular}

FT3 - free triiodothyronine; FT4 — free thyroxine; TSH — thyroid-stimulating hormone; iPTH — intact parathyroid hormone; Ca — calcium; $\mathrm{P}$ - phosphorus; ALP — alkaline phosphatase; CTX-1 — C-terminal telopeptide of type 1 collagen; P1NP — N-terminal propeptide of type $1 \mathrm{~N}$ procollagen; BMD — bone mineral density. Variables were presented as means \pm standard deviations. * significantly different $(p<0.05)$ from control group

Table 2. A comparison of the prevalence of low bone mineral density (BMD) between subjects with differentiated thyroid cancer (DTC) and controls

\begin{tabular}{|c|c|c|c|}
\hline Diseases/Quantity (percent) & DTC group $(n=65)$ & Control group $(n=50)$ & Statistical significance \\
\hline \multirow{4}{*}{ Low BMD } & \multirow{4}{*}{$29(44.62 \%)$} & \multirow{4}{*}{$14(28 \%)$} & $\chi^{2}=4.736$ \\
\hline & & & $p=0.030$ \\
\hline & & & $\mathrm{OR}=2.406$ \\
\hline & & & $95 \%$ Cl: 1.083-5.345 \\
\hline
\end{tabular}

$\mathrm{OR}$ - odds ratio; $\mathrm{Cl}$ - confidence interval

Table 3. Correlation coefficients (r values) between serum thyroid hormones levels and T-scores of bone mineral density (BMD) and bone turnover markers (BTMs) in subjects with differentiated thyroid cancer (DTC)

\begin{tabular}{lccc}
\hline Variables & FT3 & FT4 & TSH \\
\hline \multirow{2}{*}{ Mean Z scores of lumbar BMD } & $r=-0.153$ & $r=-0.156$ & $r=0.141$ \\
& $\mathrm{p}=0.221$ & $\mathrm{p}=0.198$ & $\mathrm{p}=0.231$ \\
\hline \multirow{2}{*}{ Mean Z scores of hip BMD } & $r=-0.095$ & $r=-0.103$ & $r=0.098$ \\
& $\mathrm{p}=0.47$ & $\mathrm{p}=0.461$ & $\mathrm{p}=0.491$ \\
\hline IPTH & $r=0.093$ & $r=0.113$ & $r=-0.221$ \\
\hline Ca & $\mathrm{p}=0.484$ & $\mathrm{p}=0.447$ & $\mathrm{p}=0.068$ \\
\hline $\mathrm{P}$ & $r=0.168$ & $r=0.093$ & $r=0.098$ \\
& $\mathrm{p}=0.182$ & $\mathrm{p}=0.484$ & $\mathrm{p}=0.493$ \\
\hline \multirow{2}{*}{ Vitamin D3 } & $r=0.132$ & $r=0.166$ & $r=-0.102$ \\
& $\mathrm{p}=0.308$ & $\mathrm{p}=0.173$ & $\mathrm{p}=0.463$ \\
\hline \multirow{2}{*}{ ALP } & $r=-0.125$ & $r=-0.096$ & $r=0.190$ \\
& $\mathrm{p}=0.387$ & $\mathrm{p}=0.464$ & $\mathrm{p}=0.131$ \\
\hline & $r=0.126$ & $r=-0.095$ & $r=-0.278$ \\
\end{tabular}


Table 3. Correlation coefficients ( $r$ values) between serum thyroid hormones levels and T-scores of bone mineral density (BMD) and bone turnover markers (BTMs) in subjects with differentiated thyroid cancer (DTC)

\begin{tabular}{|c|c|c|c|}
\hline Variables & FT3 & FT4 & TSH \\
\hline \multirow{2}{*}{ CTX-1 } & $r=0.209$ & $r=0.152$ & $r=-0.363$ \\
\hline & $p=0.089$ & $p=0.298$ & ${ }^{*} p=0.002$ \\
\hline \multirow{2}{*}{ P1NP } & $r=0.198$ & $r=0.215$ & $r=-0.127$ \\
\hline & $p=0.10$ & $p=0.073$ & $p=0.440$ \\
\hline
\end{tabular}

FT3 — free triiodothyronine; FT4 — free thyroxine; TSH — thyroid-stimulating hormone; iPTH — intact parathyroid hormone; Ca — calcium; P — phosphorus;

ALP — alkaline phosphatase; CTX-1 — C-terminal telopeptide of type 1 collagen; P1NP $-\mathrm{N}$-terminal propeptide of type $1 \mathrm{~N}$ procollagen; ${ }^{*}$ The $\mathrm{p}$-value at $<0.05 \mathrm{was}$ considered as statistically significant

Table 4. Serum thyroid-stimulating hormone (TSH) levels in association with T-scores of bone mineral density (BMD) and bone turnover markers (BTMs) using multivariate logistic regression

\begin{tabular}{lccc}
\hline Variables & p-value & OR & $\mathbf{9 5 \%} \mathbf{C I}$ \\
\hline Mean Z-scores of lumbar BMD & 0.121 & 1.098 & $1.105-4.245$ \\
\hline Mean Z-scores of hip BMD & 0.083 & 1.419 & $1.412-3.473$ \\
\hline iPTH & 0.159 & 1.003 & $1.045-1.493$ \\
\hline Ca & 0.179 & 1.142 & $1.259-5.317$ \\
\hline P & 0.428 & 1.001 & $1.033-2.756$ \\
\hline Vitamin D3 & 0.317 & 1.018 & $1.153-3.628$ \\
\hline ALP & $* 0.016$ & 1.432 & $0.998-6.619$ \\
\hline CTX-1 & ${ }^{*} 0.023$ & 1.323 & $0.953-3.728$ \\
\hline P1NP & 0.073 & 1.006 & $0.993-2.284$ \\
\hline
\end{tabular}

OR — odds ratio; $\mathrm{Cl}$ — confidence interval; $\mathrm{PTH}$ — intact parathyroid hormone; Ca — calcium; P — phosphorus; ALP — alkaline phosphatase; CTX-1 — C-terminal telopeptide of type 1 collagen; P1NP $-\mathrm{N}$-terminal propeptide of type $1 \mathrm{~N}$ procollagen. * The $\mathrm{p}$-value at $<0.05$ was considered statistically significant

and BTMs. Serum TSH levels, in associations with Zscores of BMD and BTMs using multivariate logistic regression in all participants, are shown in Table 4.

\section{Discussion}

The purpose of this study was to investigate the impact of long-term TSH suppression treatment on BMD and BTMs in premenopausal women with thyroid cancer after total or near total thyroidectomy. In this study, we selected iPTH, P1NP, CTX-1, Ca, P, vitamin D3, and ALP as evaluation indices of BTMs.

The effect of TSH suppression therapy on BMD in premenopausal subjects who underwent thyroidectomy remains controversial. The majority of studies suggested no effect of TSH suppression therapy on BMD in premenopausal women and men [25-27]. In this study, we failed to find a change in BMD in these premenopausal women with long-term TSH suppression therapy. The main reason is probably that premenopausal women are not at high risk for reduced bone density due to the effects of oestrogen. Oestrogen plays an important role in the growth and maturation of bone as well as in the regulation of bone formation and turnover in adult bone [28].
We demonstrated that in premenopausal women with DTC who had received at least one year of TSH suppression treatment, serum P1NP, CTX-1, and ALP levels were higher compared with controls. TSH suppression therapy in DTC patients after thyroidectomy can induce subclinical hyperthyroidism, especially in the first two years; the serum TSH levels are required to be less than $0.1 \mathrm{mIU} / \mathrm{L}$. Elevated thyroid hormones can stimulate bone turnover and bone resorption, thus leading to reduced bone strength, destruction of bone structure, and increased risk of fractures [29]. In this study, increased serum levels of P1NP, CTX-1, and ALP in DTC patients mean increased bone turnover and decreased bone quality.

In this study, we found higher prevalence of low BMD among premenopausal DTC women who received long-term LT4 therapy. Osteoporosis is less common in premenopausal women than in postmenopausal women. The main reason is the protective effect of oestrogen in premenopausal women who are less likely to develop osteoporosis. The WHO and International Society for Clinical Densitometry (ISCD) suggest the use of the $\mathrm{Z}$ scores of BMD rather than $\mathrm{T}$ scores in the diagnosis of low BMD, and the diagnosis of osteoporosis in premenopausal women is secure only when 
there is a history of low trauma fracture [30]. Some large population-based cohort studies showed that the risk of osteoporosis and fracture increased in patients receiving TSH suppression therapy. They also found that the high cumulative doses of levothyroxine were significantly associated with an increased risk of osteoporosis and fracture [31,32]. However, these conclusions were all based on postmenopausal women.

Meanwhile, we found that the serum TSH levels were negatively associated with CTX-1 and ALP, but not with BMD. These results showed that TSH suppressive therapy only increased bone turnover to a certain extent. Moreover, the degree of TSH inhibition was correlated to the degree of changes of bone turnover. The previous data of suppressive doses of levothyroxine therapy on bone mass and bone turnover in premenopausal women are limited. However, some studies on hypothyroid premenopausal women with long-term levothyroxine therapy showed increased bone turnover and increased resorptive changes, but not osteoporosis [33-35]. Hyperthyroid patients also have serum BTMs concentrations significantly higher in comparison with euthyroid subjects, in relation to thyroid hormones excess and high bone turnover ${ }^{[36]}$. Therefore, it is of great significance to evaluate the bone metabolism status in premenopausal patients with thyroid cancer after receiving long-term levothyroxine therapy, which can prevent osteopaenia or osteoporosis in the future through intervention treatment.

One limitation of our study was that the sample size was small. A larger cohort is needed to investigate if the serum TSH levels in premenopausal women receiving long-term levothyroxine treatment correlate with BMD and BTMs. In different periods after thyroid cancer surgery, there are different standards for the control of serum TSH levels [37]. Moreover, despite clinical practice guidelines for the management of DTC, there are no recommendations on rational serum TSH levels to prevent cancer recurrences and improve survival ${ }^{11}$. Therefore, in future studies, groups can be divided according to serum TSH levels, so as to more accurately judge the impact of TSH inhibition therapy on changes of bone metabolism.

\section{Conclusions}

We found a high prevalence of low BMD among premenopausal DTC women who received long-term LT4 therapy after thyroidectomy. In these patients, serum TSH levels were negatively associated with CTX-1 and ALP. Long-term TSH suppression therapy was a significant risk factor for decreased bone strength, mainly by increasing bone turnover.

\section{Conflicts of interest}

All authors have no conflicts of interest to declare in relation to this article.

\section{Funding agency}

This study received funding support from the Health Commission of Heilongjiang Province (Research project NO. 2018-091; NO. 2016-504).

\section{References}

1. Cabanillas ME, McFadden DG, Durante C. Thyroid cancer. Lancet. 2016; 388(10061): 2783-2795, doi: 10.1016/S0140-6736(16)30172-6, indexed in Pubmed: 27240885 .

2. Lim H, Devesa SS, Sosa JA, et al. Trends in Thyroid Cancer Incidence and Mortality in the United States, 1974-2013. JAMA. 2017; 317(13): 1338-1348, doi: 10.1001/jama.2017.2719, indexed in Pubmed: 28362912.

3. Mirian C, Grønhøj C, Jensen DH, et al. Trends in thyroid cancer: Retrospective analysis of incidence and survival in Denmark 1980-2014. Cancer Epidemiol. 2018; 55: 81-87, doi: 10.1016/j.canep.2018.05.009, indexed in Pubmed: 29852396.

4. Daniels GH. Follicular Thyroid Carcinoma: A Perspective. Thyroid. 2018; 28(10): 1229-1242, doi: 10.1089/thy.2018.0306, indexed in Pubmed: 30039751.

5. Cancer Genome Atlas Research Network. Integrated genomic characterization of papillary thyroid carcinoma. Cell. 2014; 159(3): 676-690, doi: 10.1016/j.cell.2014.09.050, indexed in Pubmed: 25417114

6. Tam S, Boonsripitayanon M, Amit M, et al. Survival in Differentiated Thyroid Cancer: Comparing the AJCC Cancer Staging Seventh and Eighth Editions. Thyroid. 2018; 28(10): 1301-1310, doi: 10.1089/thy.2017.0572, indexed in Pubmed: 30141373.

7. Schmidbauer B, Menhart K, Hellwig D, et al. Differentiated Thyroid Cancer-Treatment: State of the Art. Int J Mol Sci. 2017; 18(6), doi: 10.3390/ijms18061292, indexed in Pubmed: 28629126.

8. Takami H, Ito Y, Okamoto T, et al. Revisiting the guidelines issued by the Japanese Society of Thyroid Surgeons and Japan Association of Endocrine Surgeons: a gradual move towards consensus between Japanese and western practice in the management of thyroid carcinoma. World J Surg. 2014; 38(8): 2002-2010, doi: 10.1007/s00268-014-2498-y, indexed in Pubmed: 24671301.

9. Biondi B, Cooper DS. Thyroid Hormone Suppression Therapy. Endocrinol Metab Clin North Am. 2019; 48(1): 227-237, doi: 10.1016/j. ecl.2018.10.008, indexed in Pubmed: 30717904.

10. Roman BR, Morris LG, Davies L. The thyroid cancer epidemic, 2017 perspective. Curr Opin Endocrinol Diabetes Obes. 2017; 24(5): 332-336, doi: 10.1097/MED.0000000000000359, indexed in Pubmed: 28692457.

11. Biondi B, Cooper DS. Benefits of thyrotropin suppression versus the risks of adverse effects in differentiated thyroid cancer. Thyroid. 2010; 20(2): 135-146, doi: 10.1089/thy.2009.0311, indexed in Pubmed: 20151821.

12. Freudenthal B, Williams GR. Thyroid Stimulating Hormone Suppression in the Long-term Follow-up of Differentiated Thyroid Cancer. Clin Oncol (R Coll Radiol). 2017; 29(5): 325-328, doi: 10.1016/j.clon.2016.12.011, indexed in Pubmed: 28043744.

13. Reverter JL, Colomé E. [Potential risks of the adverse effects of thyrotropin suppression in differentiated thyroid carcinoma]. Endocrino Nutr. 2011; 58(2): 75-83, doi: 10.1016/j.endonu.2010.09.007, indexed in Pubmed: 21247815.

14. Williams GR, Bassett JHD. Thyroid diseases and bone health J Endocrinol Invest. 2018; 41(1): 99-109, doi: 10.1007/s40618-017-0753-4, indexed in Pubmed: 28853052.

15. Bassett JH, Williams GR. Role of thyroid hormones in skeletal development and bone maintenance. Endocr Rev. 2016; 37(2): 135-187, doi: 10.1210/er.2015-1106, indexed in Pubmed: 26862888

16. Tsourdi E, Lademann F, Siggelkow H. [Impact of thyroid diseases on bone]. Internist (Berl). 2018; 59(7): 661-667, doi: 10.1007/s00108-018-0436-Z, indexed in Pubmed: 29744522

17. Baliram R, Sun Li, Cao J, et al. Hyperthyroid-associated osteoporosis is exacerbated by the loss of TSH signaling. J Clin Invest. 2012; 122(10): 3737-3741, doi: 10.1172/JCI63948, indexed in Pubmed: 22996689.

18. Donangelo I, Suh SeY. Subclinical Hyperthyroidism: When to Consider Treatment. Am Fam Physician. 2017; 95(11): 710-716, indexed in Pubmed: 28671443.

19. Ross DS, Burch HB, Cooper DS, et al. 2016 American Thyroid Association Guidelines for Diagnosis and Management of Hyperthyroidism and Other Causes of Thyrotoxicosis. Thyroid. 2016; 26(10): 1343-1421, doi: 10.1089/thy.2016.0229, indexed in Pubmed: 27521067. 
20. de Melo TG, da Assumpção LV, Santos Ad, et al. Low BMI and low TSH value as risk factors related to lower bone mineral density in postmenospausal women under levothyroxine therapy for differentiated thyroid carcinoma. Thyroid Res. 2015; 8: 7, doi: 10.1186/s13044-015-0019-1, indexed in Pubmed: 26097507.

21. Wang LY, Smith AW, Palmer FL, et al. Thyrotropin suppression increases the risk of osteoporosis without decreasing recurrence in ATA lowand intermediate-risk patients with differentiated thyroid carcinoma. Thyroid. 2015; 25(3): 300-307, doi: 10.1089/thy.2014.0287, indexed in Pubmed: 25386760.

22. Kim CW, Hong S, Oh SeH, et al. Change of Bone Mineral Density and Biochemical Markers of Bone Turnover in Patients on Suppressive Levothyroxine Therapy for Differentiated Thyroid Carcinoma. J Bone Metab. 2015; 22(3): 135-141, doi: 10.11005/jbm.2015.22.3.135, indexed in Pubmed: 26389089.

23. Rossini M, Adami S, Bertoldo F, et al. Guidelines for the diagnosis, prevention and management of osteoporosis. Reumatismo. 2016; 68(1): 1-39, doi: 10.4081/reumatismo.2016.870, indexed in Pubmed: 27339372.

24. World Medical Association. World Medical Association Declaration of Helsinki: ethical principles for medical research involving human subjects. JAMA. 2013; 310(20): 2191-2194, doi: 10.1001/jama.2013.281053, indexed in Pubmed: 24141714.

25. Reverter JL, Holgado S, Alonso N, et al. Lack of deleterious effect on bone mineral density of long-term thyroxine suppressive therapy for differentiated thyroid carcinoma. Endocr Relat Cancer. 2005; 12(4): 973-981, doi: 10.1677/erc.1.01072, indexed in Pubmed: 16322336.

26. Yoon BH, Lee $\mathrm{Y}, \mathrm{Oh} \mathrm{HJ}$, et al. Influence of Thyroid-stimulating Hormone Suppression Therapy on Bone Mineral Density in Patients with Differentiated Thyroid Cancer: A Meta-analysis. J Bone Metab. 2019; 26(1): 51-60, doi: 10.11005/jbm.2019.26.1.51, indexed in Pubmed: 30899725.

27. Moon JH, Jung KY, Kim KM, et al. The effect of thyroid stimulating hormone suppressive therapy on bone geometry in the hip area of patients with differentiated thyroid carcinoma. Bone. 2016; 83: 104-110, doi: 10.1016/j.bone.2015.10.015, indexed in Pubmed: 26518742.

28. Cauley JA. Estrogen and bone health in men and women. Steroids. 2015; 99(Pt A): 11-15, doi: 10.1016/j.steroids.2014.12.010, indexed in Pubmed: 25555470.
29. Zhang P, Xi H, Yan R. Effects of thyrotropin suppression on lumbar bone mineral density in postmenopausal women with differentiated thyroid carcinoma. Onco Targets Ther. 2018; 11: 6687-6692, doi: 10.2147/OTT S171282, indexed in Pubmed: 30349302.

30. Cohen A. Premenopausal Osteoporosis. Endocrinol Metab Clin North Am. 2017; 46(1): 117-133, doi: 10.1016/j.ecl.2016.09.007, indexed in Pubmed: 28131128

31. Melton LJ, Ardila E, Crowson CS, et al. Fractures following thyroidectomy in women: a population-based cohort study. Bone. 2000; 27(5): 695-700, doi: 10.1016/s8756-3282(00)00379-3, indexed in Pubmed: 11062358 .

32. Lin SY, Lin CL, Chen HT, et al. Risk of osteoporosis in thyroid cancer patients using levothyroxine: a population-based study. Curr Med Res Opin. 2018; 34(5): 805-812, doi: 10.1080/03007995.2017.1378174, indexed in Pubmed: 28884595.

33. Babu RP, Christy A, Hegde A, et al. Do premenopausal hypothyroid women on levothyroxine therapy need bone status monitoring? Clin Med Insights Womens Health. 2015; 8: 1-6, doi: 10.4137/CMWH.S22114, indexed in Pubmed: 25861238.

34. Baqi L, Payer J, Killinger Z, et al. Thyrotropin versus thyroid hormone in regulating bone density and turnover in premenopausal women. Endocr Regul. 2010; 44(2): 57-63, indexed in Pubmed: 20429634.

35. Abrahamsen Bo, Jørgensen HL, Laulund AS, et al. The excess risk of major osteoporotic fractures in hypothyroidism is driven by cumulative hyperthyroid as opposed to hypothyroid time: an observational register-based time-resolved cohort analysis. J Bone Miner Res. 2015; 30(5): 898-905, doi: 10.1002/jbmr.2416, indexed in Pubmed: 25431028.

36. Amato G, Mazziotti G, Sorvillo F, et al. High serum osteoprotegerin levels in patients with hyperthyroidism: effect of medical treatment. Bone. 2004; 35(3): 785-791, doi: 10.1016/j.bone.2004.04.021, indexed in Pubmed: 15336617.

37. Haugen BR, Alexander EK, Bible KC, et al. 2015 American Thyroid Association Management Guidelines for Adult Patients with Thyroid Nodules and Differentiated Thyroid Cancer: The American Thyroid Association Guidelines Task Force on Thyroid Nodules and Differentiated Thyroid Cancer. Thyroid. 2016; 26(1): 1-133, doi: 10.1089/thy.2015.0020, indexed in Pubmed: 26462967. 